\title{
Statistical Evaluation of Diagnostic Tests
}

\author{
Vishnu Vardhan Rudravaram
}

\begin{abstract}
The use of routine laboratory tests in diagnosing disease is becoming of increasing importance. This emphasizes to test the efficiency of diagnostic tests, since relatively few diagnostic tests correctly classify all subjects tested as diseased or well. The more usual situation is one in which some well subjects are classified as diseased and some diseased subjects classified as well. In this type of situation, Diagnostics and prognostic models serve the purpose. Diagnostic models are usually used for classification and quite commonly used in medical field. In this paper, importance of statistical classification procedures are highlighted which helps in the evaluation of diagnostic tests.
\end{abstract}

Keywords--- Classification Procedures, Diagnostic Tests, Biomarkers, ROC Curve

\section{INTRODUCTION}

$\mathrm{I}$ $\mathrm{N}$ the last two decades, the assessment of diagnostic tests has been rapidly increasing. A test procedure has been proposed for the evaluation of single diagnostic test and an extensive study and gave a test statistic for the comparison and evaluation of two diagnostic tests [10], [13]. An outline about the statistical treatment which helps in handling the problems with respect to one or more diagnostic procedures has been discussed [6]. Further an extensive review on the statistical techniques used for medical decision making, confining to the applications in diagnostic radiology [12]. Statistical Decision Making techniques in medicine are useful in determining the importance of the diagnostic tests, identification of threshold levels and so on. The primary function of a diagnostic test is to rule in or rule out disease, in turn which gives rise to the meaning that relatively few diagnostic tests correctly classify all individuals tested as diseased or well. The usual situation is one in which some well individuals are classified as diseased and some diseased individuals classified as well. To make a perfect decision making on the diagnostic tests there is a need to use advanced statistical techniques such as Decision Analysis, Regression Analysis, Cost-Effective Analysis, Logistic Regression, Receiver Operating Characteristic (ROC) curves, Discriminant Analysis and many more.

In this paper, importance of statistical classification procedures are highlighted, which helps in evaluating the diagnostic test, an attempt is also made to provide a discriminant score cutoff which enables to classify the new individual into one of the two groups. In the next section, we brief out the concepts of ROC curve analysis and Discriminant Analysis.

Vishnu Vardhan Rudravaram, Assistant Professor, Department of Statistics, Pondicherry University, Puducherry, India. E-mail: rvvcrr@gmail.com

\section{ROC CURVE AND ITS MEASURES}

The ROC curve has its mathematical formulation which helps in fitting and estimating the parameters of the curve. The entire classification is carried out on the basis of a threshold value often referred to as Gold Standard, it determines the true condition status and also provides a source of information completely different from the tests under evaluation. The true condition status indicates the presence of disease otherwise absence. Two basic measures of ROC curves are sensitivity $(\mathrm{Sn})$ and specificity $(\mathrm{Sp})$.

Sensitivity refers to the ability of a test to detect the condition when it is present and Specificity refers to the ability of test to exclude the condition in patients without the condition.

An ROC curve is a plot of $1-\mathrm{Sp}$ versus $\mathrm{Sn}$. The construction of ROC curve primarily depends on the four possible states which are obtained on the basis of a threshold value i.e., TP, TN, FN and FP. The resulting curve is called empirical ROC [7]. Conventionally, it is assumed that diseased (D) and the healthy $(\mathrm{H})$ individuals follow Normal distribution and hence the name Binormal ROC curve, with unknown monotonic transformation [1], [3]. Another important measure in ROC curve analysis is the Area Under the Curve (AUC), it is referred as the best index of discrimination [7], [8]. Basing on the AUC value the performance of a diagnostic test can be interpreted and it takes its range from 0.5 to 1.0. Higher the AUC values, the better will be the performance of a diagnostic test. If, for a test AUC $<0.5$, one should not consider that test for further classification. A theoretical work has been proposed by combining the recent variable selection methods with AUC methods to optimize diagnostic accuracy of multiple risk factors [15]. The robustness of using the bi-normal model to fit the ROC curves [14]. A General linear model approach has been proposed for evaluating the ROC form and as well as estimating the AUC [11]. A linear regression frame work has been introduced to evaluate the ROC model by involving the covariate effects on the ROC curves [5].

\section{PARAMETRIC FORM OF ROC CURVE}

Let $\mathrm{D} \sim \mathrm{N}(\mu \mathrm{D}, \sigma \mathrm{D} 2)$ and $\mathrm{Y} \sim \mathrm{N}(\mu \mathrm{H}, \sigma \mathrm{H} 2)$, where $\mathrm{D}$ represents data from patients with disease and $\mathrm{H}$ represents data from those without disease. We can define false-positive and true-positive rates as

$$
\begin{aligned}
& F P(c)=1-P(X>c)=1-\Phi\left[\frac{c-\mu_{H}}{\sigma_{H}}\right]=\Phi\left[\frac{\mu_{H}-c}{\sigma_{H}}\right] \\
& \text { and } \\
& \operatorname{TP}(\mathrm{c})=\mathrm{P}(\mathrm{X}>\mathrm{c})=\Phi\left[\frac{\left(\mu_{\mathrm{D}}-c\right)}{\sigma_{\mathrm{D}}}\right]
\end{aligned}
$$


where $\mathrm{c}$ is the cutoff decision point and $\Phi$ is the cumulative normal distribution function.

The ROC curve can be traced out by the functions FP and TP as [4]

$$
[\mathrm{FP}(\mathrm{c}), \operatorname{TP}(\mathrm{c})]=\left\{\Phi\left[\frac{\mu_{H^{-}} \mathrm{c}}{\sigma_{\mathrm{H}}}\right], \Phi\left[\frac{\mu_{\mathrm{D}}-\mathrm{c}}{\sigma_{\mathrm{D}}}\right]\right\}-\infty<\mathrm{c}<\infty
$$

The expression of area under the ROC curve is given as [3]

$$
\mathrm{AUC}=\Phi\left[\frac{\mathrm{a}}{\sqrt{1+\mathrm{b}^{2}}}\right]
$$

here $a=\frac{\mu_{D}-\mu_{H}}{\sigma_{D}}$ and $b=\frac{\sigma_{H}}{\sigma_{D}}$.

\section{DISCRIMINANT ANALYSIS}

Discriminant analysis is a technique where the discrimination is done by linear composites. A function that separates objects may sometimes serve as an allocator and one that allocates objects may sometimes act as discriminator. Using this technique, the discrimination function can be obtained which helps in classification of new individuals or objects into one of the groups. This technique helps in identifying the group centroids of various groups considered using which a cutoff can be obtained.

Let Xi $(\mathrm{i}=1,2, \ldots . . \mathrm{m})$ be the variable from $\mathrm{Np}(\mu \mathrm{i}, \Sigma \mathrm{i})$ with ni samples each. Assume that $\Sigma 1=\Sigma 2=\Sigma 3=\ldots \ldots . . \mathrm{m}$. The optimality of classification depends on the assumptions of data which are

1. The predictor variables follow multivariate normal distribution.

2. The covariance matrices of different groups of data are homogeneous.

The discriminant function is nothing but a linear combination of the variables in the study. It is given as

$$
D=\beta_{0}+\beta_{1} x_{1}+\beta_{2} x_{2}+\cdots+\beta_{m} x_{m}
$$

where $\mathrm{D}$ is the discriminant score. This score is used to evaluate the function and evaluation is done using probabilities. The value of $\mathrm{D}$ is found out from the above expression by substituting the sample points in it. Thus, a set of $\mathrm{n}$ discriminant scores can be obtained. The correlation between the $D$ values and $x_{j}$ values can be computed which explains the measure of contribution of the $j^{\text {th }}$ variable in discriminating the groups. However, we cannot obtain fruitful information if $x_{j}$ and $x_{1}(j \neq 1)$ are highly correlated with each other.

Let the populations under study be denoted as $\Pi 1$ and $\Pi 2$. The classification rule can be made functional and the object is placed under П1 [2] if

$$
\begin{aligned}
& {\left[\left(\bar{X}_{1}-\bar{X}_{2}\right)^{\prime} S_{\text {pooled }}^{-1}\right] x-\frac{1}{2}\left(\bar{X}_{1}+\bar{X}_{2}\right)^{\prime} S_{\text {pooled }}^{-1}\left(\bar{X}_{1}+\bar{X}_{2}\right) \geq} \\
& \quad \ln \left[\left(\frac{c(1 \mid 2)}{c(2 \mid 1)}\right)\left(\frac{p_{2}}{p_{1}}\right)\right]
\end{aligned}
$$

otherwise the object is assigned to $\Pi 2$. Here $\overline{\mathrm{X}}_{1}$ and $\overline{\mathrm{X}}_{2}$ are unbiased estimates of $\mu 1$ and $\mu 2$, respectively and Spooled is the unbiased estimate of $\Sigma$. c(1|2) and $\mathrm{c}(2 \mid 1)$ are the costs of misclassification. $\mathrm{p} 1$ and $\mathrm{p} 2$ are prior probabilities of $\Pi 1$ and
П2 respectively. Here the $\mathrm{x}$ value is the new observation which has to be classified into one of the groups.

\section{RESULTS AND DISCUSSIONS}

Since the ADA values are normally distributed in both Healthy and Diseased groups, it is likely that the Binormal ROC produces more accurate AUC.

The fitted ROC model is found to be ROC $=\Phi(\mathrm{z})$, where $\mathrm{z}$ $=6.5483+3.0546 * \Phi-1(1-\mathrm{Sp})$.

It means that the $\mathrm{Sn}$ value is the cumulative standard normal probability corresponding to $\mathrm{z}$. The linear function $\mathrm{z}$ is a measure of discriminating ability of the marker. The intercept estimates the standardized gap between the means of the two groups while the slope estimates the scale with which $\mathrm{z}$ increases.

The AUC is estimated as 0.9792. This is lower than the AUC found from the non-parametric method which gave AUC $=1.00$. One possible reason for this is that the data in each group contained some extreme values which have influenced the parameters $\mathrm{a}$ and $\mathrm{b}$ of the Binormal model.

The outliers are removed and the entire calculations are performed again. The following section shows the comparative details of the results.

\section{i. $\quad$ Modified Results when Outliers are removed}

\begin{tabular}{|c|c|c|c|}
\hline $\begin{array}{l}\text { S. } \\
\text { No }\end{array}$ & Characteristics & With Outliers & Without Outliers \\
\hline 1 & $\mathrm{n}_{1}$ & 33 & 23 \\
\hline 2 & $\mathrm{n}_{2}$ & 67 & 57 \\
\hline 3 & $\begin{array}{c}\text { - K-S Test: H } \\
\text { Group } \\
\text { - K-S Test: D } \\
\text { Group }\end{array}$ & $\begin{array}{l}Z=0.824 ; \\
p-\text { value }=0.506 \\
Z=0.669 ; \\
p \text {-value }=0.763\end{array}$ & $\begin{array}{l}Z=0.694 ; \\
p \text {-value }=0.721 \\
Z=0.601 ; \\
\text { p-value }=0.863\end{array}$ \\
\hline 4 & Trimmed Mean & $\begin{array}{l}\text { H-Group: } 20.61 \\
\text { D-Group: } 74.51\end{array}$ & $\begin{array}{l}\text { H-Group: } 20.56 \\
\text { D-Group: } 73.97\end{array}$ \\
\hline 5 & $\begin{array}{ll}\text { - } & \text { AUC: } \\
& \text { Nonparametr } \\
& \text { ic } \\
\text { - } & \text { AUC: } \\
& \text { Binormal } \\
\end{array}$ & $\begin{array}{l}1.00 \\
0.979\end{array}$ & $\begin{array}{l}1.00 \\
0.997\end{array}$ \\
\hline 6 & $\begin{array}{ll} & \text { Mean } \pm \text { S.D } \\
& \text { for H Group } \\
\text { - } & \text { Mean } \pm \text { S.D } \\
& \text { for D Group } \\
\end{array}$ & $\begin{array}{l}20.48 \pm 8.519 \\
76.27 \pm 26.022\end{array}$ & $\begin{array}{l}20.65 \pm 6.678 \\
74.07 \pm 18.086\end{array}$ \\
\hline 7 & Optimal Cutoff & $36 \mathrm{IU} / \mathrm{L}$ & $36 \mathrm{IU} / \mathrm{L}$ \\
\hline 8 & $\mathrm{~S}_{\mathrm{n}}$ & $1.00(100 \%)$ & $1.00(100 \%)$ \\
\hline 9 & $\left(1-S_{p}\right)$ & $0.00(0.00 \%)$ & $0.00(0.00 \%)$ \\
\hline 10 & $\begin{array}{l}\text { Binomial } \\
\text { Parameters }\end{array}$ & $\begin{array}{l}\mathrm{a}=6.5483 ; \mathrm{b}= \\
3.0546\end{array}$ & $\begin{array}{l}a=7.9986 ; b= \\
2.7081\end{array}$ \\
\hline
\end{tabular}

When the outliers are removed the data set is left out with $\mathrm{n} 1=23$ cases in H-group and $\mathrm{n} 2=57$ cases in D-group. The following results provide a comparison between the two data sets, with outliers and without outliers.

It can be observed that removal of the outliers has resulted in higher AUC in case of Binormal ROC, while there is no change in the nonparametric AUC. The Binormal model had a better fit when outliers are removed. The standardized 
difference between means of diseased and healthy groups is measured by the parameter ' $a$ ', where as ' $b$ ' indicates the standard deviation of diseased group relative to healthy group. By removing the outliers the mean distance between populations is better identified when compared to the case with outliers. Again, removal of outliers has lead to lower relative standard deviation.

The Binormal ROC curve with and without outliers is shown in Figure 1

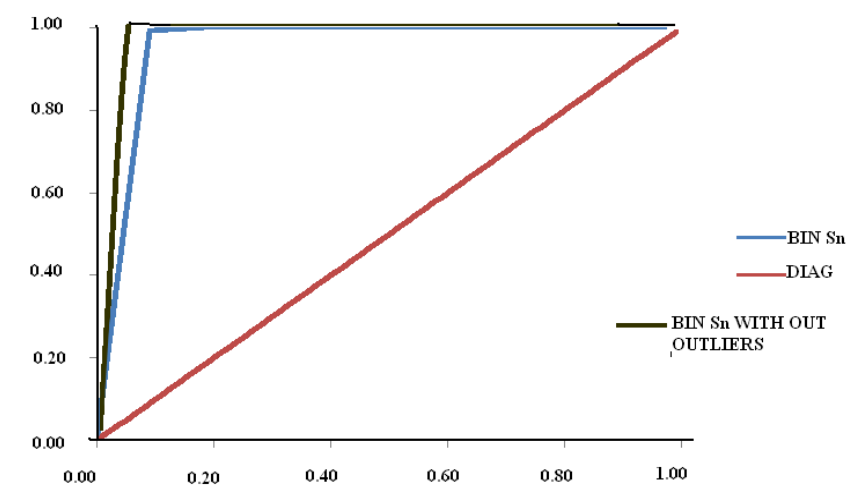

Figure 1: Binormal ROC Curves with and Without Outliers

It can be seen that, when the outliers are removed the ROC curve shows better shape.

\section{ii. $\quad$ Effect of Age and Sex as Cofactors}

The effect of cofactors like age and sex along with ADA can be brought into a Linear Discriminant Function (LDF) frame work so that a new patient subject with unknown group membership can be classified into TB / Non-TB group with a reasonable level of accuracy.

Using the data (Outliers removed) the following LDF is obtained with all variables included in the model (Enter Method).

$\mathrm{D}_{\mathrm{i}}=-3.081+0.062 * \mathrm{ADA}-0.023 * \mathrm{AGE}+0.433 * \mathrm{SEX}$

where Di is the discriminant score for the ith subject.

When this function is evaluated at the group means of the explanatory variables, we get the following scores.

\begin{tabular}{|l|c|}
\hline \multicolumn{1}{|c|}{ Group } & $\begin{array}{c}\text { Function score } \\
\text { at the centroids (means) }\end{array}$ \\
\hline TB group & 1.040 \\
\hline Non TB group & -2.577 \\
\hline Average (cutoff) & -0.7685 \\
\hline
\end{tabular}

Now for the ith subject we evaluate the discriminant scores Di using (7). If Di $\leq-0.7685$, classify the subject as Non-TB otherwise classify as TB. These discriminant scores and the group membership are saved in the SPSS file as new variables. Since the actual scores are also known, the discriminating ability of the LDF is given by the confusion matrix which gives the percentage of correct classifications. This LDF correctly classifies $97.5 \%$ of the subjects. The following graph shows the ROC curve when the LDF given in (7) is used as a classifier. The AUC is found to be 0.9589 .

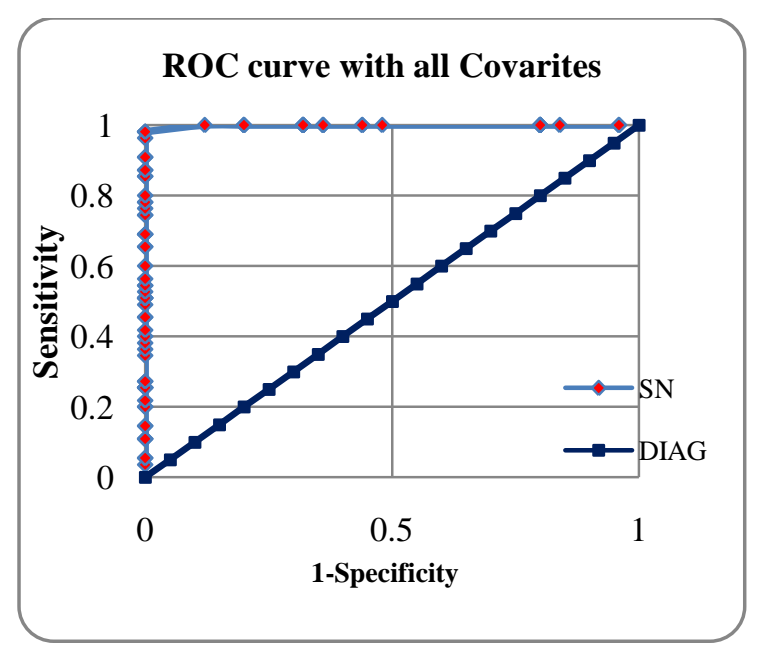

Figure 2: ROC Curve with all COVARIATES

Suppose AGE and SEX are not included in the model, then the discrminant function becomes

$\mathrm{D}=-3.733+0.064 *$ ADA

The cutoff score for discrimination is -0.722 and the following table of misclassification is observed. This method has given $96.3 \%$ of correct classification.

\section{iii. Stepwise Method of Estimating the LDF}

Suppose LDF is estimated using stepwise regression instead of full regression, then the most important variables alone will be in the model and we get the following results.

1. Stepwise Linear Discriminant Function $\mathrm{D}_{\mathrm{i}}=-2.935+0.063 * \mathrm{ADA}_{\mathrm{i}}-0.020 * \mathrm{AGE}_{\mathrm{i}}$

(8)

2. Function value at the group centroids and the cutoff

\begin{tabular}{|l|c|}
\hline \multicolumn{1}{|c|}{ Group } & $\begin{array}{c}\text { Function score } \\
\text { at the centroids (means) }\end{array}$ \\
\hline TB group & 1.019 \\
\hline Non TB group & -2.525 \\
\hline Average (cutoff) & -0.753 \\
\hline
\end{tabular}

3. It is observed that this LDF correctly classifies $98.8 \%$ of the subjects.

4. The ROC curve for this LDF is shown in Figure (3)

5. The AUC of this LDF is 1.00

6. The Distribution of discrminant scores in each of the two groups is shown in Figure (4) 


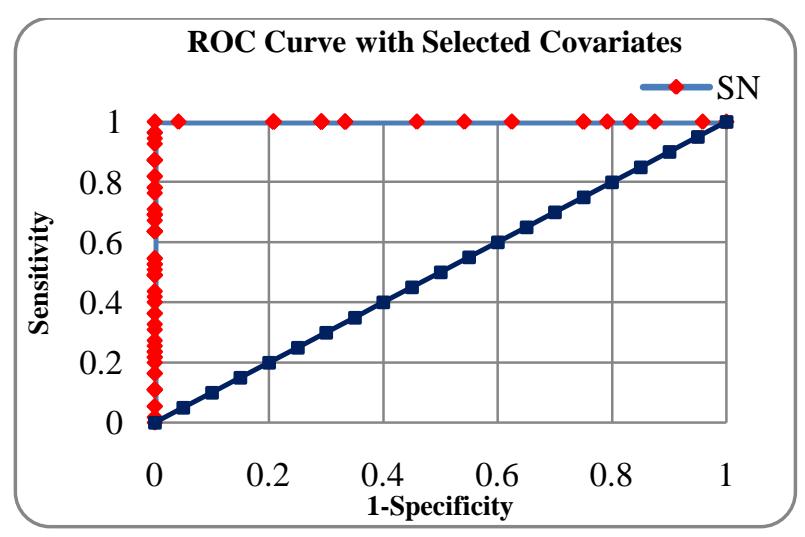

Figure 3: ROC Curve with Selected Covariates

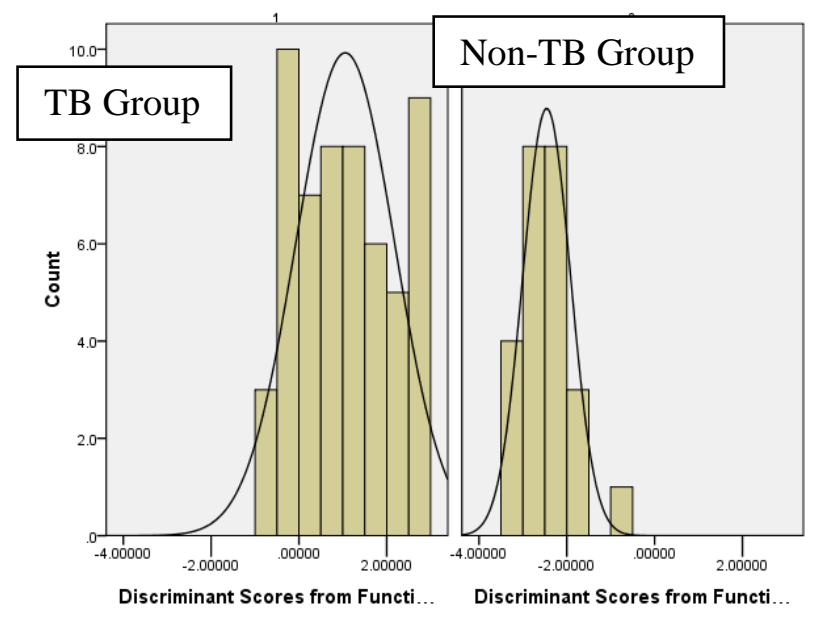

Figure 4: Distribution of Discriminant Scores in the TB and Non-TB Groups

It can be seen that the stepwise linear discriminant function offers a better classification of subjects into correct groups using pleural $\mathrm{ADA}$ and $\mathrm{AGE}$ as the determinants of TB status.

This result helps in the screening of patients with unknown TB status. The ROC curve of the stepwise LDF has an AUC = 1.00 , which means nearly $100 \%$ correct classification is done. The percentage of correct classification with different methods is shown below.

\begin{tabular}{|l|c|}
\hline Method & $\begin{array}{c}\text { Cases Correctly } \\
\text { Classified }\end{array}$ \\
\hline Full regression with ADA & $96.3 \%$ \\
\hline $\begin{array}{l}\text { Full regression with ADA, } \\
\text { AGE and SEX }\end{array}$ & $97.5 \%$ \\
\hline $\begin{array}{l}\text { Stepwise regression with ADA, } \\
\text { AGE }\end{array}$ & $98.8 \%$ \\
\hline
\end{tabular}

It is also observed that the percentage of correct classification has increased with different discriminant functions.

\section{CONCLUSIONS}

In this paper, focus was made on highlighting the importance of diagnostic test and the implications of statistical techniques in evaluating the diagnostic tests. ROC curve analysis has been carried out to identify the model and the accuracy measure for a diagnostic test. Further, different discriminant functions were identified with various cofactor combinations. For each cofactor combination, discriminant function and discriminant scores were obtained. The average value of discriminant scores of healthy and diseased groups was considered as optimal cutoff point. Further, this discriminant score cutoff can be used to classify a new individual into one of the two known groups. It is proposed that for further classification the discriminant score cutoff can be used instead of clinical cutoff.

\section{REFERENCES}

[1] Bamber, D. The area above the ordinal dominance graph and the area below the receiver operating characteristic graph. Journal of Mathematical Psychology, 12: 387-415, 1975.

[2] Carl J Huberty, Applied Discriminant Analysis, John Wiley and Sons, 1994

[3] David Farragi and Benjamin Reiser, Estimation of the area under the ROC curve, Statistic in Medicine; 21; 3090-3106, 2002

[4] Donna Katzman Mc Clish, Analyzing a Portion of the ROC curve , Medical Decision Making 1989; 9; 190-195, 1989.

[5] Hanley, J A, The Robustness of the Binormal Assumption used in fitting ROC curves, Medical Decision Making; 8: 197 - 203, 1988

[6] J. Neyman, Outline of Statistical Treatment of the Problem of Diagnosis, Public Helath Reports, 62, 40, 1947.

[7] James A Hanley, Barbara J Mc Neil, A Meaning and use of the area under a receiver Operating Characteristics (ROC) curves, Radiology; $143 ; 29-36,1982$.

[8] James A Hanley, Barbara J Mc Neil, A method of Comparing the Areas Under Receiver Operating Characteristics Analysis derived from the same cases, Radiology; 148; 839-843, 1983.

[9] Krazonowski W and Hand D J, ROC curves for Continuous Data , Chapman and Hall, 2009.

[10] Nathan Mantel, Evaluation of a Class of Diagnostic Tests, Biometrics, 240-246, 1951.

[11] Pepe M.S. An interpretation for the ROC curve and inference using GLM procedure. Biometrics, 56, 352-359, 2000.

[12] Peter M. Doubilet, Statistical Techniques for Medical Decision Making: Applications to Diagnostic Radiology, American Journal of Radiology 150:745-750, 1988.

[13] Samuel W. Greenhouse and Nathan Mantel, The Evaluation of Diagnostic Tests, Biometrics, 399-412, 1950.

[14] Zhang, Z., \& Pepe M.S. A linear regression Framework for Receiver Operating Characteristic (ROC) curve analysis. The Berkely Electronic Press, Pp., 253, 2005

[15] Zhou, XH., Chen, B., Xie, Y.M., Tian, F., Liu, H., \& Liang, X. Variable selection using the optimal ROC curve: An application to a traditional Chinese medicine study on osteoporosis disease. Statistics in Medicine, DOI:10.1002/sim.3908, 2012.

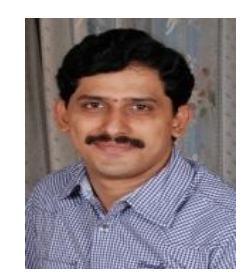

$\mathrm{R}$ Vishnu Vardhan is currently working as Assistant Professor in the Department of Statistics, Pondicherry University, Puducherry. His areas of research are Biostatistics and Statistical Computing. $\mathrm{He}$ has published 11 research papers in reputed journals and participated. He has organized one national workshop. He has presented 25 research papers in 18 National and International conferences/Seminars, $\mathrm{He}$ is a recipient of Ms Bhargavi Rao and Padma Vibhushan Prof. C R Rao Award for best Poster Presentation in an International Conference and also recipient of Indian Society for Probability and Statistics (ISPS) Young Statistician Award during December 2011. He is a life member of Indian Society for Probability and Statistics. He has written a book entitled "One Some of Statistical Methods for Clinical Trials - A Study of ROC Curves" 